\title{
Evaluation of Histogram of Oriented Gradients Soft Errors Criticality for Automotive Applications
}

\author{
FERNANDO FERNANDES, LUCAS WEIGEL, CLAUDIO JUNG, PHILIPPE NAVAUX, \\ LUIGI CARRO, and PAOLO RECH, Federal University of Rio Grande do Sul
}

Pedestrian detection reliability is a key problem for autonomous or aided driving, and methods that use Histogram of Oriented Gradients (HOG) are very popular. Embedded Graphics Processing Units (GPUs) are exploited to run HOG in a very efficient manner. Unfortunately, GPUs architecture has been shown to be particularly vulnerable to radiation-induced failures. This article presents an experimental evaluation and analytical study of HOG reliability. We aim at quantifying and qualifying the radiation-induced errors on pedestrian detection applications executed in embedded GPUs.

We analyze experimental results obtained executing HOG on embedded GPUs from two different vendors, exposed for about 100 hours to a controlled neutron beam at Los Alamos National Laboratory. We consider the number and position of detected objects as well as precision and recall to discriminate critical erroneous computations. The reported analysis shows that, while being intrinsically resilient (65\% to $85 \%$ of output errors only slightly impact detection), HOG experienced some particularly critical errors that could result in undetected pedestrians or unnecessary vehicle stops.

Additionally, we perform a fault-injection campaign to identify HOG critical procedures. We observe that Resize and Normalize are the most sensitive and critical phases, as about $20 \%$ of injections generate an output error that significantly impacts HOG detection. With our insights, we are able to find those limited portions of HOG that, if hardened, are more likely to increase reliability without introducing unnecessary overhead.

CCS Concepts: • Computer systems organization $\rightarrow$ Reliability; $\bullet$ Hardware $\rightarrow$ Transient errors and upsets; $\bullet$ General and reference $\rightarrow$ Reliability;

Additional Key Words and Phrases: HOG, pedestrian detection

\section{ACM Reference Format:}

Fernando Fernandes, Lucas Weigel, Claudio Jung, Philippe Navaux, Luigi Carro, and Paolo Rech. 2016. Evaluation of histogram of oriented gradients soft errors criticality for automotive applications. ACM Trans. Archit. Code Optim. 13, 4, Article 38 (November 2016), 25 pages.

DOI: http://dx.doi.org/10.1145/2998573

\section{INTRODUCTION}

Thanks to their low cost, increased energy efficiency, and flexible development platforms, low-power embedded Graphics Processing Units (GPUs) have enjoyed widespread adoption in various application domains, including the automotive one. Embedded GPUs, in fact, are part of projects implementing the Advanced Driver Assistance Systems, which analyzes camera or radar signals to detect pedestrians or obstacles and activate the car brakes to prevent collisions [European New Car Assessment Programme 2012]. Additionally, autonomous driving systems, which is the new trend in the automotive market, rely on humans or obstacles computer-aided

Authors' addresses: Universidade Federal do Rio Grande do Sul, Bloco IV, Avenida Bento Gonçalves, 9500 Agronomia, Porto Alegre - RS, 91509-900; emails: \{ffsantos, lfweigel, crjung, navaux, carro, prech\}@inf. ufrgs.br.

Permission to make digital or hard copies of part or all of this work for personal or classroom use is granted without fee provided that copies are not made or distributed for profit or commercial advantage and that copies show this notice on the first page or initial screen of a display along with the full citation. Copyrights for components of this work owned by others than ACM must be honored. Abstracting with credit is permitted. To copy otherwise, to republish, to post on servers, to redistribute to lists, or to use any component of this work in other works requires prior specific permission and/or a fee. Permissions may be requested from Publications Dept., ACM, Inc., 2 Penn Plaza, Suite 701, New York, NY 10121-0701 USA, fax +1 (212) 869-0481, or permissions@acm.org.

(c) 2016 ACM 1544-3566/2016/11-ART38 $\$ 15.00$

DOI: http://dx.doi.org/10.1145/2998573 
detection. The reliability characterization of algorithms that implement objects detection is then mandatory to ensure automotive systems safety. We specifically consider the Histograms of Oriented Gradients (HOG) algorithm [Dalal and Triggs 2005], which is the core of several pedestrian or object detection systems [Sivaraman and Trivedi 2013; Zhu et al. 2006; Kang and Lim 2014; Li and Guo 2013]. HOG can be combined with a variety of classifiers to detect pedestrians. In this work, we choose to use HOG as a feature and Support Vector Machine (SVM) as a classifier [Dalal and Triggs 2005]. If otherwise stated, then in the rest of the article we address the conjunction of HOG and SVM simply as HOG.

Various sources of errors could undermine the system reliability, including environmental perturbations, software errors, and process, temperature, or voltage variations [Lutz 1993; Laprie 1995; Nicolaidis 1999; Baumann 2005]. The generated error may corrupt data values or logic operations and lead to Silent Data Corruption (SDC), Application Crash, and System Hang or be masked and cause no observable error [Constantinescu 2002; Saggese et al. 2005; Schroeder et al. 2009]. We focus on radiationinduced soft errors that, according to Baumann [2005], are a huge concern in modern computing devices because, uncorrected, they produce a failure rate that is higher than all the other reliability mechanisms combined. In fact, today the radiation-induced failure rate represents a significant issue not only in radiation-harsh environments, such as space, but also in milder environments at sea level. Due to the shrinking of transistor dimensions and the exacerbation of the amount of available resources, electronic devices are becoming more susceptible to soft errors induced by ionizing particles. High-energy neutrons generated by the interaction of cosmic rays with the terrestrial atmosphere may then have enough energy to corrupt data stored in static randomaccess memories or to affect logic computations [Baumann 2005]. Radiation-induced failures are especially relevant in safety-critical applications, for which reliability is mandatory.

While extremely efficient in terms of FLOP/s (floating-point operations per second) and FLOPs-per-WATT, modern GPUs have been shown to be prone to experience radiation-induced corruption [DeBardeleben et al. 2013; Wunderlich et al. 2013; Gomez et al. 2014; Oliveira et al. 2014; de Oliveira et al. 2016]. GPU architecture may be particularly susceptible to be corrupted by radiation for three main reasons. (1) GPUs were traditionally designed for entertainment video editing or gaming applications, for which reliability is not a concern [Breuer et al. 2004]. Thus, their architecture is optimized to increase performance more than reliability. (2) GPUs orchestrate parallel processes using hardware scheduler and dispatcher. To make parallel executions more efficient, GPUs use shared memory among threads. The corruption of the scheduler or an error in the shared memory is likely to affect the computation of several parallel processes, significantly lowering the GPU reliability [Rech et al. 2013]. (3) GPUs require a huge amount of memory to implement parallelism, both caches and register files. These memories have been demonstrated to be the cause of the majority of errors in modern computing devices [Seifert et al. 2002; Tan et al. 2011]. While Central Processing Unit (CPU) cores include parity, Error Correcting Code (ECC) or other error protection mechanisms on their main memory structures even in commercial Systems On Chip (SoCs) [AMD 2015; ARM 2016; Intel 2016], most embedded GPUs, including the ones considered in this article, still lack any protection. As an example, Tesla's self-diving system is powered by NVIDIA Tegra K1 [Nvidia 2014], which does not have any reliability solution applied to the GPU core. As shown in this article, the lack of architectural hardening makes embedded GPUs prone to experience soft errors when applied in safety-critical systems. It is worth noting that the latest GPUs for High Performance Computing applications include ECC on the main memory structures [NVIDIA 2015; Intel 2014], which has been shown to reduce of about one order of magnitude the GPU error rate [de Oliveira et al. 2016]. Lately, some SoCs with 
ECC-protected GPU memories have appeared in the market [Nvidia 2016; AMD 2015] but are not employed in any self-driving car yet. As discussed in Section 5, ECC is likely to improve significantly SoCs reliability and reduce software hardening overhead.

To experimentally evaluate the reliability of HOG executed on embedded GPUs, we take advantage of the control neutron beam available at the Los Alamos National Laboratory (LANL) Los Alamos Nuclear Science Center (LANSCE) facility, in Los Alamos, New Mexico. We exposed six embedded GPUs from two different vendors to the neutron flux for about 100 hours of experiment. As the LANSCE beam is suitable to mimic the terrestrial one, we can consider errors observed at LANSCE as representative of errors that can occur on realistic HOG utilization [Violante et al. 2007].

Our analysis goes a step beyond the traditional SDCs detection by considering error criticality. Besides measuring the number of radiation-induced output errors we also identify four metrics, each providing unique information on the effect of observed SDCs on the pedestrian detection reliability. We consider the number of pedestrians detected, the center of mass of the detection, and Precision and Recall. Correlating the selected metrics, it is possible to identify those critical errors that could prevent a pedestrian detection or erroneously lead the vehicle to a sudden stop.

Finally, we perform a fault-injection campaign to identify those HOG portions whose corruption is likely to affect pedestrian detection in a critical way. Protecting only the algorithm portions that have been identified as the causes for critical errors will result in an efficient and effective hardening for HOG. It is worth noting that in cost-sensitive domains, such as the automotive sector, where efficiency in terms of per-unit-prices is a key criterion, full hardware redundancy or dedicated hardware modifications are to be considered too expensive. Moreover, in real-time systems, full time redundancy overhead may impede to meet deadlines. Our analysis serves as a reference for the design of efficient and effective selective hardening solutions.

The main contributions of the article are as follows: (1) the experimental evaluation of the behavior of HOG executed on embedded GPUs exposed to radiation, (2) a publicly accessible repository with the observed radiation-induced errors [dos Santos et al. 2016], (3) the utilization of formal metrics to describe the radiation-induced errors on pedestrian detection systems and to evaluate their criticality, (4) the identification of the most vulnerable HOG procedures through fault-injection, and (5) concrete proposals on how to increase HOG and embedded GPUs reliability for automotive applications.

The remainder of the article is organized as follows. Section 2 gives a background on HOG structure and reviews functional safety for automotive applications concepts. Section 3 presents the proposed error criticality metrics, Section 4 describes the adopted experimental methodology, Section 5 presents and discusses experimental and faultinjection results, while Section 6 concludes the article.

\section{BACKGROUND AND RELATED WORK}

This section serves as a background to understand HOG importance and the main steps the algorithm performs to identify pedestrians. The algorithm analysis is also necessary to understand the causes of observed errors and select the critical procedures to be hardened. Then, functional safety for automotive systems is discussed.

\subsection{Histogram of Oriented Gradients}

In this work, we evaluate the reliability and error criticality of HOG included in the OpenCV (Open Source Computer Vision) library, which is an open-source computer vision library for C++, C, Python, and Java languages [Bradski 2000]. The OpenCV HOG version is optimized for automotive applications, and both CUDA and OpenCL versions are available.

HOG is one of the most common features for pattern or object detection [Yang et al. 2012; Lim et al. 2013; Ren and Ramanan 2013], particularly in automotive 


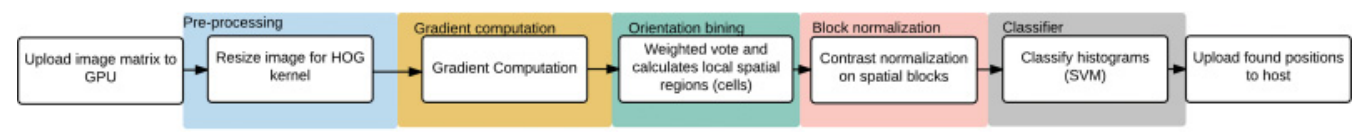

Fig. 1. OpenCV HOG algorithm execution flow. Adapted from Dalal and Triggs [2005].

applications [Sivaraman and Trivedi 2013], and its application on both human [Zhu et al. 2006; Kang and Lim 2014] and vehicle [Li and Guo 2013] detection are widespread on today's self-driving vehicles. One of the reasons for HOG popularity is normalization, which eliminates illumination variance on daylight scenes, improving shape detection. HOG orientation sampling is extremely versatile and can be defined to detect different objects. By simply changing the classifier characteristic, HOG can be used to detect humans or objects as well as to recognize faces or gestures.

HOG can be combined with a variety of classifiers to detect pedestrians. In this work, we choose to use HOG as a feature and SVM as a classifier. This choice was made for two main reasons: (1) HOG combined with SVM is one of the most popular and effective detection systems [Dalal and Triggs 2005; Dollar et al. 2012] and (2) the availability of HOG together with SVM on a publicly accessible library eases the reproduction of our results [Bradski 2000]. We anticipate that while radiation experiments results are strictly related to the chosen feature and classifier, the reliability discussion and fault-injection results for HOG are directly extendable to other classifiers.

In the chosen pedestrian detector, each frame is analyzed by creating a set of Bounding Boxes (BBs), which are pedestrian candidates. Within these BBs, the features are extracted and classified using an SVM, yielding a set of validated BBs. Figure 1 shows the OpenCV HOG behavior. The steps shown in the picture are separately analyzed in the following. The fault-injection routine described in Section 4.4 is used to identify which step is more critical. Results are presented in Section 5.3.

HOG is basically a feature descriptor code designed for objects detection. The CPU triggers the execution loading to the GPU, the image matrix, and the data of a previously trained SVM. The method is composed of five GPU steps: Gradient computation, Orientation binning, Descriptor Blocks and Block normalization, and Classifier [Dalal and Triggs 2005]. The OpenCV version of HOG has a special feature over the original method: an image pre-processing for color correction (see Figure 1). Before performing HOG main steps, the GPU executes a lookup texture on the processing frame.

Gradient Computation is the first step, in which a simple derivative mask filter is applied to the image to detect gradients (i.e., edges). The kernel is a very simple mask applied to every pixel on the image and, as such, intrinsically robust against soft errors. In fact, an error in a step of a filter is averaged with correct values and typically does not significantly affect the output. This is in accordance with our fault-injection results in Section 5.3, which demonstrate that this step is particularly robust and hard to be the cause of observed errors.

The second phase of HOG is Orientation binning. In this phase, the image is divided into a fixed number of $8 \times 8$ pixels regions called cells. A histogram of gradient orientations is computed on the pixels within each cell. The histogram is calculated with a weighted vote on the cell, which is a function of the gradient magnitude at the pixel [Dalal and Triggs 2005].

The Descriptor Blocks and Block normalization phase groups adjacent cells as spatial regions, called blocks, of various dimensions. Cells are grouped based on their gradient orientation, and a cell can participate to more than one block. Once cells are grouped, their histograms are normalized to avoid illumination and contrast bias. A cell can have different normalizations, one for each block it belongs to. Each block is represented by a block descriptor, which is a vector that considers the contributions 
of all the normalized cells in the block. The final object (i.e., a potential BB) detection is based on various blocks descriptors. Blocks typically overlap so each block may contribute to various objects. The next step of HOG classifies objects into BBs.

The peculiarity of cells to participate to various blocks and of the object to be formed of various blocks could significantly impact HOG behavior under radiation. We claim that it is more likely for a radiation-corruption to affect HOG in a way that increases, rather than decreases, the number of BBs. Let us assume that a block (or the descriptor of a block) that participates to a BB got corrupted in a way that prevents HOG to consider it as part of the object. As blocks overlap, HOG could still identify the object without the corrupted block thanks to other correctly computed blocks. For HOG to miss a BB, radiation should corrupt most of the blocks that compose that $\mathrm{BB}$, which is unlikely. On the other hand, there are two situations in which radiation can induce additional BBs. (1) The corruption of a block that is part of a BB leads HOG to create an additional BB over the same portion of the image or to split the BB into two BBs. (2) The corruption of a block that should not be part of a BB leads HOG to create an additional BB over a portion of the image that does not represent a human (or object). This latter situation may lead HOG to create a BB with the dimension of a single block or to erroneously group the faulty block with adjacent ones to create a larger BB. Metrics presented in Section 3 evaluate the probability of additional or missing BBs to occur, and the results presented in Section 5.2 confirm our hypothesis. Additionally, the adopted metrics allow us to evaluate the criticality of additional or missing BBs for automotive applications. As demonstrated in Section 5.2, additional BBs mostly overlap on the same pedestrian.

The last step of the HOG method is Classifier. The soft linear SVM classifier is trained with SVMLight, which solves several problems on SVM vectors classifiers [Joachims 1999]. Humans (or other objects) are identified by BBs, described by four coordinates. A different training could be used to identify other objects in the image. This step is particularly critical for the overall system reliability. We anticipate that errors in the matrix that results from the SVM training significantly impact pedestrian detection (details in Section 5.3).

\subsection{Functional Safety for Automotive Systems}

One of the most important features in self-driving cars is human (or obstacles) detection. From 2016, only vehicles implementing pedestrian detection are eligible to receive 5 stars (which is the highest achievable score) in the security evaluation from the European New Car Assessment Program (Euro NCAP), which is the most authoritative car safety evaluation agency in Europe [European New Car Assessment Programme 2012]. In this scenario, it is fundamental to test detection algorithms and validate their reliability.

To be applied in automotive applications, any system must be compliant with the strict ISO26262 constraints [Dongarra et al. 2015]. For safety-critical automotive applications, like autonomous driving or pedestrian detection, the requirements are identified with Automotive Safety Integrity (ASIL) level D, which is the highest classification of injury risk. ASIL level D is actually the most stringent level of safety measures to be applied to avoid an unreasonable residual risk. It imposes any component of the system to be able to detect $99 \%$ of permanent and transient faults. The allowed hardware failure probability (either radiation related or not) is limited to 10 Failures In Time (FIT, i.e., errors in $10^{9}$ hours of operations).

Such a requirement is extremely strict. To have a reference, let us measure the FIT rate considering as errors the fatal crashes that occurred in the United States in 2013, which The National Highway Traffic Safety Administration (NHTSA) estimates to have been 30,057 . Of those, 10,076 were driver-related fatal crashes that we can assume would have been avoided with a reliable self-driving or crash warning system. 
There were 5,657,000 non-fatal crashes were [Dongarra et al. 2013]. To measure the FIT rate, beside the number of events, it is necessary to calculate the number of hours of operations (i.e., the total number of hours driven in 2013). For NHTSA, the number of Vehicle Miles Traveled in 2013 was about $3 \times 10^{12}$. If we conservatively assume that those miles were traveled with an average speed that ranges between 25 and 50 Miles Per Hour (MPH), then we conclude that accidents occurred in a time window between $12 \times 10^{10}$ and $6 \times 10^{10}$ hours. If we consider the fatal crashes caused by direct responsibility of the driver as failures that occurred in the measured time window, then we have a fatal crash FIT rate between 84 and 168. For non-fatal crashes, the FIT rate would result in about 28,582. As stated, the ASIL level D target is 10 FIT. Having self-driving cars fully compliant with ISO26262 would reduce the current fatal crash rate from 9 to 17 times and the non-fatal crash rate more than 3,000 times [Saxena 2016]. This evaluation on one side demonstrates the importance of reliable autonomous cars and, on the other, highlights the challenging effort required to be compliant with ISO 26262. Unfortunately, today's self-driving cars are still far from reaching the goal of 10 FIT. Google reported its first car accident caused by system error in February 2016 [Google 2016b]. Considering that the Google cars have driven about $1.5 \times 10^{6}$ miles until June 2016 (as reported in Google [2016a]) their FIT rate, under the assumption of an average speed between 25 and 50MPH, is between 16,000 and 33,000 FIT, well above the ASIL-D requirements.

\section{ERROR CRITICALITY EVALUATION}

In this section, we present the metrics we claim to be necessary to analyze HOG corrupted output criticality. Traditionally, the radiation-induced error rate is calculated considering SDCs and Crashes as failures. Any result produced by the tested device that deviates from the expected one is marked as erroneous and considered in failure rate calculation. Our intention is to go beyond the traditional bit-per-bit comparison between the experimental output and the radiation-free output, that is, the expected value for HOG output. We select four metrics that derive from image processing community to qualify HOG radiation-induced corruptions: Diff BBs, Center of Mass, Precision, and Recall. The importance of each metric on the reliability evaluation of HOG depends on the application or on the system in which HOG is applied. As described in Section 5.2, some metrics are particularly important to identify critical errors and give essential insights to the device or application designers to improve the reliability of their product.

\section{Diff BBs}

The Diff BBs metric represents the difference between the expected number of BBs and the number of BBs in the radiation-corrupted output. As discussed in Section 2.1, a $\mathrm{BB}$ symbolizes a pedestrian identification, which is the main information provided by HOG. Diff BBs then provides a first evaluation of the radiation impact of pedestrian detection, and it is essential to describe radiation-induced effects on the algorithm. The Diff BBs value is obtained subtracting the number of BBs contained in the radiationfree execution from the number of BBs contained in the corrupted output. Diff $B B s<0$ means that radiation corrupted $\mathrm{HOG}$ in a way that reduces the number of $\mathrm{BBs}$, while Diff_BBs $>0$ states that radiation-induced corruption led to additional BBs.

It is not possible to state $a$ priori that Diff_BBs $=0$ is sufficient or necessary to ensure that HOG corruption was not critical, that is, detection is still fine. In fact, on one side Diff_BBs $=0$ only states that the number of BBs in the output is correct, but it does not give any information about their shape or position in the picture. On the other side, Diff_BBs $\neq 0$ is not sufficient to mark the error as critical. In fact, additional BBs may overlap with others making the correlation more $B B s=$ more area detected not 
necessarily true. Additionally, missing BBs may be covered by bigger ones, refuting the correlation that Diff $B B s<0$ implies that there are undetected portions of the image that should have been detected. In both cases (Diff_BBs $>0$ and Diff_BBs $<0$ ), the correct number of pedestrians could still have been detected.

To have deeper insights on output criticality, Center of Mass and Precision and Recall metrics are presented that aim at evaluating the distribution of BBs in the picture, the portion of the picture covered by the corrupted output, and the similarity of the produced output and the result from a fault-free environment.

\section{Center of Mass}

Given the distribution of BBs in the picture, we can determine the coordinates of the pixel that represent their center of mass. To evaluate how the corruption changed BBs' overall disposition (size and position), we plot the difference between the experimental output Center of Mass and the radiation-free output Center of Mass. A positive value of Center of Mass in the $x$-axis indicates that the radiation-induced error causes BBs to lean towards the right side of the image, while a negative value indicates that BBs Center of Mass are erroneously shifted to the left side of the image. The same applies to the $y$-axis, with positive values indicating that Center of Mass is shifted towards the top of the image.

\section{Precision and Recall}

In the image processing community, Precision and Recall are widely used to compare the BBs identified by the algorithm with the ground truth to access the quality of a given classifier [Fawcett 2006]. To evaluate how radiation affects HOG execution, it is necessary to employ Precision and Recall to compare the experimental output with the radiation-free output (not with the ground truth). This choice is dictated by the goal of this article, which is to evaluate the radiation impact on HOG and analyze how it modifies HOG behavior. For radiation experiment purposes, it is sufficient (and necessary) to execute HOG on a single frame (details in Section 4.2). Calculating Precision and Recall of the corrupted output with respect to the ground truth (and not with the radiation free output) would depend on the selected frame, as HOG detection quality does. This latter calculation, while interesting, would not be exhaustive and would not reveal precise HOG reliability issues.

Precision and Recall are given by:

$$
\begin{gathered}
\text { Precision }=\frac{T P}{T P+F P}, \\
\text { Recall }=\frac{T P}{T P+F N},
\end{gathered}
$$

where $T P$ is the number of True Positives (pedestrians that were actually detected), $F P$ is the number of False Positives (outcomes of the classifier that do not correspond to a pedestrian), and $F N$ is the number of False Negatives (a pedestrian that was not detected by the classifier). To qualify experimental results, we consider a BB $n$ in the radiation-corrupted output as TP if for any $\mathrm{BB} m$ of the radiation-free output the following condition is verified:

$$
\text { Jaccard_similarity }(n, m)>T_{J},
$$

where $T_{J}$ is the acceptance threshold. Otherwise, we consider $n$ as an FP. If, for a given $\mathrm{BB} m$ of the radiation-free output, there is no $\mathrm{BB} n$ on the corrupted output that satisfies this condition, then an $\mathrm{FN}$ is detected.

$T_{J}$ is an arbitrary threshold, with $0 \leq T_{J} \leq 1$. Values of $T_{J}$ close to 1 impose the classifier to be extremely precise, that is, BBs detected by HOG have to match the 
ground truth exactly. Values of $T_{J}$ closer to 0 relax detection precision. $T_{J}=0.5$ has been identified as a good tradeoff to evaluate detection quality, as it allows some detection imperfection yet maintaining a good relation with the ground truth [Fawcett 2006]. When evaluating the impact of radiation on HOG, $T_{J}$ becomes a threshold to distinguish between critical and noncritical corruptions. Any corruption that preserves Equation (3) is not to be considered critical, as BBs are to be considered sufficiently similar to the radiation-free output. When Equation (3) is not verified, it means that radiation induces an $\mathrm{FP}$ or $\mathrm{FN}$ (i.e., additional or missed pedestrian, respectively). As such, $T_{J}=1$ imposes any radiation-induced error to be marked as critical while values of $T_{J}$ close to 0 allow most corruptions to be acceptable. In the reliability context, $T_{J}=0.5$ still holds, as the $T_{J}$ tradeoff discussion presented in Fawcett [2006] is valid independently from the source of detection imprecision (intrinsic algorithm detection imprecision or radiation-induced corruption). Then, to compare HOG radiation corrupted output with the radiation-free output we select $T_{J}=0.5$.

The Recall rate provides the fraction of existing pedestrians that were actually detected by the classifier, even in the event of radiation-induced error. Hence, $R=100 \%$ means that all pedestrians were successfully detected. On the other hand, Precision measures the fraction of the detections produced by the classifier that actually relate to a pedestrian, so $P=100 \%$ means that all detections produced by the classifier correspond to pedestrians. In general, the detection imprecision (either radiation related or not) emphasizes Precision at the expense of Recall or vice versa. An error could then lower Recall (i.e., miss some pedestrians) but increase Precision (i.e., reduce number of false positives) or the other way around. The $F$-score combines Precision and Recall values by measuring their harmonic mean. In applications where either low Recall is preferable to low Precision or vice versa, the $F_{\beta}$ score is more adequate. $F_{\beta}$ is given by

$$
F_{\beta}=\left(1+\beta^{2}\right) \frac{\text { Precision } \cdot \text { Recall }}{\beta^{2} \text { Precision }+ \text { Recall }},
$$

where $\beta>0$ is a weight. To assign a higher weight to Recall, one should select $\beta>1$, whereas $\beta<1$ prioritizes Precision. In the context of pedestrian detection for automotive applications, low Recall rates may result in undetected pedestrians while low Precision rates could lead to unnecessary sudden stops.

The presented metrics allow one to fully analyze the criticality of errors in the produced output: Diff_BBs quantifies the radiation-induced effects on HOG output and Center of Mass analyses BBs distribution. Precision and Recall qualify the impact of radiation on pedestrian detection: Recall measures missed pedestrians while Precision measures portions of the image erroneously tagged as pedestrians.

\section{EXPERIMENTAL METHODOLOGY}

In this work, we have opted to evaluate the reliability of HOG executed on embedded GPUs through accelerated radiation testing. Then, we use fault injection to detect critical portions of both the feature and the classifier.

Both radiation experiments and fault-injection information is required for the scopes of this work. The gathering of realistic data is essential to precisely evaluate the radiation reliability of HOG executed on embedded GPUs and analyze the criticality of radiation-induced errors. Such an evaluation, for those devices like Commercial Off-The-Shelf (COTS) products for which a Register Transfer Level (RTL) description is not available, can be performed only through radiation experiments. In fact, without an RTL description, faults can be injected only on a selection of user-accessible resources, while radiation testing does not restrain faults to a single part of the chip. Additionally, fault-injection results could be biased on the selected fault model while radiation experiments mimic the realistic probabilities and manifestations 
of failures, as described in the Radiation Hardness Assurance (RHA) testing procedure [Herrera-Alzu and Lopez-Vallejo 2014].

While offering a plethora of advantages, radiation experiments provide a very limited error propagation analysis. It is very hard to correlate SDCs or Crashes with a set of resources or algorithm procedures whose corruption lead to the observed failure. We use a fault-injector to identify HOG's critical procedures, as we intend to deeply analyze its criticality and provide insights for designing efficient hardening solutions. The intrinsic limitations of fault-injection on COTS make it impossible to correlate each physical radiation-induced error with its manifestation at the output. However, by corrupting variable values, we are able to identify those parts of the code that are likely to affect HOG execution [Hari et al. 2014]. In other words, we can calculate the SDC rate for HOG, which is the percentage of injected errors that caused SDCs. Additionally, as discussed in Section 5.3, the correlation of fault-injection and radiationexperiment outputs allows us to identify which procedures or variables are more likely to generate the observed critical failures and, thus, should be hardened.

This section describes the tested GPUs and selected HOG configurations. Then, the adopted setups for radiation experiments and fault injection are detailed.

\subsection{Devices Under Test}

We select two commercially available embedded GPU architectures for our studies, which in this article are called Platform A and Platform B. The choice of anonymity was made not to reveal business-sensitive information of our industrial partners.

Platform A: This architecture includes four CPU cores, designed with an operating frequency of up to $4.1 \mathrm{GHz}$. The CPU has $256 \mathrm{~KB}$ of L1 data and instruction cache and $4 \mathrm{MB}$ of $\mathrm{L} 2$ cache. The general processor power consumption is about $95 \mathrm{~W}$. The Platform A's embedded GPU has eight cores with an operating frequency of about $866 \mathrm{MHz}$. Platform A's main memory is DDR3 with a frequency of $2.133 \mathrm{MHz}$, using at most two channels. The GPU can execute up to 512 parallel threads, and the CPU up to 4 threads.

Platform B: This embedded SOC is powered by an ARM quad-core Cortex-A15 from the ARMv7 family, with an operating frequency of $2.3 \mathrm{GHz}$. The ARM core disposes of $32 \mathrm{~KB}$ of $\mathrm{L} 1$ data and instruction cache and $2 \mathrm{MB}$ shared L2 cache. The embedded GPU has 192 computing cores with $64 \mathrm{~KB}$ of register file. As main memory, Platform B disposes of $2 \mathrm{~GB}$ of DD3L at $933 \mathrm{MHz}$.

Neither Platform A nor Platform B GPU cores offer any reliability solution like ECC. Both Platform A and Platform B are designed with a Single Instruction Multiple Data (SIMD) architecture. SIMD processors allows a single instruction to operate on a group of data in parallel [Hennessy and Patterson 2011], commonly arrays and matrices. Since modern embedded GPUs can provide hundreds of processing units, it is easy to achieve great speedups on Computer Vision applications that require a massive use of operations on arrays and matrices, like HOG.

\subsection{Applications Under Test}

For our experimental and analytical study, we use the Dalal and Triggs HOG method, implemented on the OpenCV library, version 2.4.9. The version we select has an opensource sample test to allow developers to set the method's parameters. We set number of levels to 100, hit threshold to 0.8, and group threshold to 1, following OpenCV directives for frames in which some objects can be covered by more than one BB [Bradski 2000].

The quality of classifiers is proved using videos from available datasets. Such proof is required as classifiers are not perfect, and their performances depend on the selected frames. Researchers are then aiming at improving the average performances of their algorithms on several representative frames. The scope of this article differs. We aim 

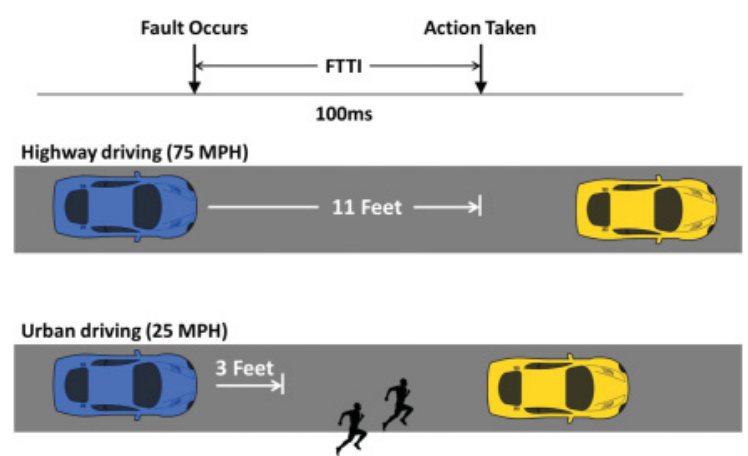

Fig. 2. Fault Tolerant Time Interval (FTTI) when driving in highway or urban context. Adapted from Saxena [2016].

at characterizing radiation impact on $\mathrm{HOG}$ execution, that is, how the number of $\mathrm{BBs}$, their position, and proprieties are affected by radiation with respect to a radiation-free execution (not with respect to the ground truth). It is worth noting that the radiationfree execution may differ from the ground truth. However, the evaluation of HOG detection quality is out of the scope of this article, as it has already been deeply investigated [Dalal and Triggs 2005].

For the aims of the proposed study, it is sufficient to run HOG on a single frame, as long as the frame is sufficiently complex and representative. In fact, as HOG is a feature descriptor, the operations performed and program flow do not depend on the image itself. In other words, each step detailed in Section 2.1 will be executed independently on the number of humans or objects in the frame. The chosen static frame is illustrated in Figure 4(a). The image is sufficiently complex to induce HOG to create several BBs of different sizes and positions and includes clusters of pedestrians, cars, and other objects. This single frame allows us then to evaluate how radiation impacts HOG detection in a wide set of situations. The chosen frame was specifically selected for radiation experiments and is not part of datasets commonly used to evaluate detection quality. This choice was dictated by the fact that frames on available data sets, while universally used to evaluate detection quality, may be too simple to evaluate HOG reliability. Testing HOG on a single frame is not only sufficient but also necessary, as radiation experiments are very time consuming and costly. Testing multiple frames would not provide a sufficient number of errors to characterize HOG corruptions in each of them.

It is also worth noting that errors affecting a single frame may significantly undermine HOG-based autonomous driving systems, even if subsequent frames are correctly analyzed. In fact, while HOG could be tuned to take the action basing its decision on several frames, there are situations in which such a solution may not be possible. The Fault Tolerant Time Interval (FTTI), defined (but not quantified) in the ISO26262 as the time between the fault occurrence and the action execution, may not be always sufficient to process several frames. Figure 2 shows two cases, one in which the car is driving on the highway at 75MPH and the other where the car is in an urban context at $25 \mathrm{MPH}$. Let us assume conservatively that, to prevent collision, the action (i.e., brakes activation) has to be taken approximatively 11 Feet in the first case or 3 Feet in the second from the detection. As 11 Feet at $75 \mathrm{MPH}$ and 3 Feet at $25 \mathrm{MPH}$ are traveled in only $100 \mathrm{~ms}$, the assumption narrows the FTTI to $100 \mathrm{~ms}$. Unfortunately, as most modern car cameras have rates of 30 Frames/sec, only 3 frames may be available for HOG to be analyzed in this interval. This restricts significantly the possibility of analyzing sequential frames. Additionally, the fault-injection we performed was used to detect 


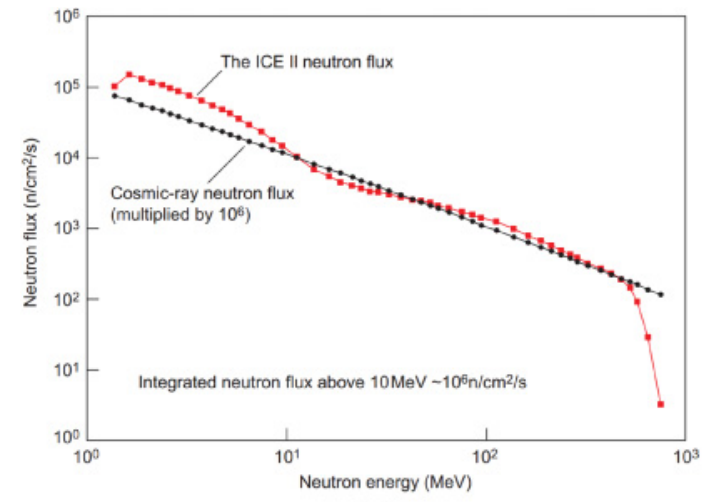

(a)

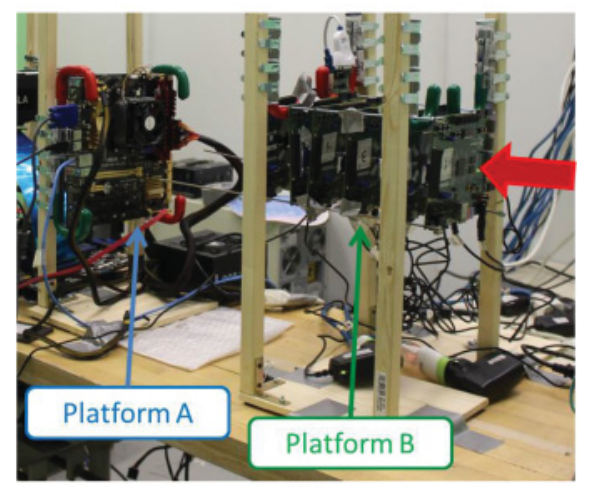

(b)

Fig. 3. (a) LANSCE neutron spectrum compared to the terrestrial one. (b) Part of the experimental setup at LANSCE. A total of 2 Platform A and 4 Platform B embedded GPUs were aligned with the neutron beam. The beam direction is indicated by the arrow.

procedures whose corruption leads to persistent failures that result in errors affecting several frames (results in Section 5.3).

\subsection{Experimental Setup}

Radiation experiments aim at measuring the cross section, which is the sensitive area of the device, that is, that portion of area that, if hit by an impinging particle, causes an observable failure. When a kernel is tested, the cross section is measured dividing the observed error rate (errors $/ s$ ) by the average particle flux (particles $/\left(\mathrm{cm}^{2} \times \mathrm{s}\right)$ ), yielding an area. The larger the cross section, the higher the use of sensitive resources [Baumann 2005; Mukherjee et al. 2003]. So, the cross section depends on the overall amount of resources required for computation and on their criticality but does not include any information on execution time.

Our experiments were performed at the ICE House II at the LANSCE facility in November 2015. As shown in Figure 3(a), LANSCE provides a white neutron source that emulates the energy spectrum of the atmospheric neutron flux between 10 and $750 \mathrm{MeV}$. As LANSCE reasonably mimics the atmospheric neutron flux, the probability of having a neutron corrupting the SoC during our experiments or at sea level during normal operation are very similar [Violante et al. 2007]. The experimentally observed cross-section gains then importance as it becomes an intrinsic characteristic of the device and application, independent on the neutron source. Multiplying the experimentally measured cross-section $\left(\mathrm{cm}^{2}\right)$ by the expected neutron flux on the SoC $\left(13 n /\left(\mathrm{cm}^{2} \times \mathrm{h}\right)\right.$ at sea level [JEDEC 2006]), one can estimate the realistic FIT rate of the device executing the application.

The neutron flux available at LANSCE was about $2.5 \times 10^{6} n /\left(\mathrm{cm}^{2} \times \mathrm{s}\right)$, about 8 orders of magnitude higher than the atmospheric neutron flux. In a realistic environment, it is highly likely to have no more than one corruption during a single execution. To maintain this behavior, experiments were tuned to guarantee observed output error rates close to $10^{-3}$ errors/execution, ensuring that the probability of more than one neutron generating a failure in a single code execution remains negligible.

The beam was restricted to a spot with a diameter of 2 inches, which was enough to fully irradiate Platform A and Platform B chips without directly affecting nearby board power control circuitry and Dynamic Random-Access Memory (DRAM) chips. To ensure that the data stored in the main memory are not corrupted, allowing an analysis 
focused on the devices' core reliability; we checked for radiation-induced errors in the DRAM and never observed any. We have aligned two Platform A and four Platform B GPUs with the beam to reduce statistical error. The chips were then placed at different distances from the neutron source. A de-rating factor was applied to consider distance attenuation. After the de-rating, the devices error rate seemed independent on the position, suggesting that the neutron attenuation caused by other boards between the source and the device under test is negligible.

Figure 3(b) shows part of the experimental setup mounted at LANSCE. A host computer initializes the test sending pre-selected input to the embedded GPU and gathers results, comparing them with a pre-computed golden output. It is worth noting that this comparision takes no more than $1 \%$ of the total execution time. When a mismatch is detected, the execution is marked as affected by an SDC.

Software and hardware watchdogs were included in the setup. The software watchdog monitors a timestamp written by the application under test. If the timestamp is not updated in a few seconds, then the kernel is killed and launched again. This watchdog detects Crashes, that is, application crashes or control flow errors that prevent the GPU from completing assigned tasks (e.g., an infinite loop). The hardware watchdog is an Ethernet controlled switch that performs a power cycle of the host computer if the host computer itself does not acknowledge any ping requests in 10 minutes. The hardware watchdog is necessary to detect Hangs.

\subsection{Fault-Injection}

We perform a fault-injection routine on several parts of the algorithm, using a procedure similar to GPU-Qin [Fang et al. 2014]. We have injected faults in main local kernel variables both on the host and GPU parts of HOG. The fault-injection procedure was performed through GNU Project debugger (GDB). The SDC and Crash detection were performed using exactly the same setup described for radiation experiments. While the main purpose of our fault-injection campaign is to better understand the observed radiation-induced errors, the derived insights could be extended to other sources of SDC. In fact, the observed HOG behaviors when faults are injected in variables does not depend on the physical source of error. Results presented in Section 5.3 can be extended to SDCs that arise from memory errors caused by capacity and temperature variations [Schroeder et al. 2009] as well as electric noises or capacitive coupling [Saggese et al. 2005] or intermittent faults caused by circuit defect on complementary metal-oxide-semiconductor microprocessors and static/dynamic memory [Constantinescu 2002].

On the GPU side, we perform separate fault-injection campaigns for the specific kernels regions of the main HOG phases (detailed in Section 2.1). Using a Python script and GDB, we are able to freeze the HOG execution flow and change local variables values on both the GPU kernels and the host side routines. Before fault-injection starts, we map all accessible local kernel variables into a list. We then choose, randomly, a line on one of the five HOG kernels (see Section 2.1) to place a GDB breakpoint. When fault-injection starts, the program runs normally until the breakpoint is reached. At the breakpoint, execution is frozen and a variable from the local kernel variables list is randomly chosen. The context is switched to the host, which performs error injection by assigning a random value to the selected variable. The breakpoint is then deleted and execution continues. When radiation generates a bit flip (or multiple bit flips) on low-level resources, the bit flip may propagate, resulting in a wrong value written to memory. From a high-level view, the wrong variable value is not necessarily limited to a single bit of difference from the correct value. As we inject faults in high-level variables, injecting random values (which includes but is not limited to single bit flips) is the fault model that better fits our purposes. Thus, we have preferred to use a random value generator and inject random values rather than inject only single bit flips. 
The fault-injection is much simpler on the CPU than on the GPU as the procedure is sequential on the host. With GDB, we are able to step into OpenCV functions and change variables' values in a random fashion.

\section{RESULTS AND DISCUSSION}

This section first reports results obtained through radiation experiments. The metrics discussed in Section 3 are applied to radiation experiment results to qualify HOG corruption criticality. Fault-injection results are then reported to identify critical procedures. Finally, we take advantage of the experimental and analytical analyses to propose hardening solutions to increment HOG reliability in embedded SoCs.

\subsection{Beam Experiments Results}

Each device was tested for an overall of about 100 hours under the beam. As discussed in Section 4.3, the LANSCE flux is about 8 orders of magnitude higher than the atmospheric neutron flux. Each device received a number of neutrons equivalent to about $10^{10}$ hours (i.e., about $10^{6}$ years) of operation in natural environment. Experimental results are available in a publicly accessible repository [dos Santos et al. 2016]. We underline that the setup detailed in Section 4.3 was designed following JEDEC guidelines [JEDEC 2006] to allow a direct downscale of experimental results to the much less intense natural neutron flux.

At each execution, the data calculated was compared bit per bit with the result of the radiation-free execution. The application execution was then classified as follows:

Correct: The application produced exactly the expected output of a fault-free environment.

SDC: The application produced a different output than that of a fault-freeenvironment. This corrupted output is stored and post-processed.

Crash: The system hanged or crashed and had to be restarted, or the system rebooted by itself.

The observed SDC error rate under the accelerated beam, considering the output as corrupted if there is any mismatch between the expected output and the computed output, was of $3.12 \times 10^{-4}$ errors/executions for Platform A and $9.34 \times 10^{-4}$ errors / executions for Platform B. While, to prevent leakage of business-sensitive data, we cannot reveal absolute FIT rates for the devices, we can say that SDC FIT rate for both Platform A and Platform B are in the order of tens of FITs, above the limit of 10 FIT imposed by ASIL-D.

The Crash rate was of $1.25 \times 10^{-3}$ crashes / executions for Platform A and $6.93 \times 10^{-3}$ crashes/executions for Platform B SoCs. It is not surprising that for both Platform A and Platform $B$ Crashes are more likely than SDCs. HOG, in fact, acts as a filter and operates image processing, which are intrinsically robust against SDCs [Breuer et al. 2004]. As a result, radiation errors that affect data memory elements or operations could be masked during the computation, not affecting the output. In other words, the SDC rate of HOG is likely to be small. The last three steps of HOG (Orientation Binning, Normalize, and Classifier) have several control flow operations whose corruption is likely to modify the algorithm flow, eventually generating infinite loops or illegal memory accesses, which lead to Crashes. It is worth noting that a significant component of Crashes is caused by radiation corruption of the device control circuitry. Errors affecting instruction memory, the GPU hardware schedulers, or the CPU-GPU interface could lead to application crash or system hang, independently of the algorithm proprieties [de Oliveira et al. 2016]. Crashes, while more frequent than SDCs in $\mathrm{HOG}$, are considered less critical as they are easily detected [Li et al. 2008; Nakka 
et al. 2005; Pattabiraman et al. 2006]. Crash detection and recovery in a real-time system, however, must be quick enough to allow the system to recover without missing deadlines [Candea and Fox 2001; Lee and Sha 2005; Wu et al. 2006].

HOG is found to be more prone to be corrupted when executed on Platform A. As discussed in Section 5.2, Platform A is also more prone to critical errors than Platform $B$. The most likely reason for the observed discrepancies relies on the number of active parallel processes, which is greater on Platform A. This means that a higher amount of resources will be shared on Platform A with respect to Platform B. A corruption on those shared resources is likely to affect several parallel processes, eventually producing a critical error. Other reasons could be the different transistors layout, different parallel processes scheduling, and different computing units designs. Unfortunately, as both Platform A and Platform B designs and characteristics are proprietary, it is not possible to exhaustively understand the reasons for the observed different behaviors under radiation.

Experiments highlighted a critical Platform B SoC configuration. Platform B relies on embedded flash memory to store the system boot-loader. Under radiation, this choice does not seem reliable. In fact, several times during our experiments, the flash memory got corrupted, impeding the correct system boot. To have Platform $B$ functionality restored, it was necessary to re-flash the embedded memory. A boot-loader corruption is detected with two subsequent activations of the hardware watchdog, which takes at least 20 minutes. This impedes a precise measure of the boot-loader FIT rate. However, FIT rates for Flash memories of several vendors and technologies are publicly available [Just et al. 2013; Bagatin et al. 2014; Fogle et al. 2004] and are to be used as a reference to estimate the error rate in realistic applications. As modern flash memories have been demonstrated not to be immune to terrestrial radiation, it is advisable not to rely only on flash memory to store safety-critical application key data.

\subsection{Error Criticality Analysis}

Figure 4 shows an example of observed radiation-corrupted output. Radiation disturbs HOG execution in a way that changes the number of identified pedestrians (BBs). Intuitively, most pedestrians identified by a radiation-free execution of HOG (Figure 4(a)) are still detected. However, there are a lot of portions of the picture that the radiationfree HOG execution did not tag, while the radiation-corrupted one did. In this section, metrics presented in Section 3 are applied to compare radiation experiment results with the radiation-free output (not with the ground truth) to evaluate errors criticality. In the following discussion, only corrupted outputs are considered, and all averages are absolute values.

We first consider Diff BBs, which is the difference between the number of Bounding Boxes (i.e., detected pedestrians) in the radiation-free output and in the corrupted output (in Figure 4 Diff_BBs $=16$ ). If radiation corrupts HOG in a way that a greater number of pedestrians is detected, then Diff BBs will be greater than zero and smaller than zero otherwise. Figure 5 shows the percentage of corrupted outputs affected by the different Diff BBs values for both Platform A and Platform B. Of radiation-induced errors, $45 \%$ did not affect the number of BBs detected in Platform B and $24 \%$ in Platform $A$ SoCs. Platform $B$ had overall smoother results, with Diff_BBs values always smaller that 4, while Platform A struggled with Diff_BBs between -7 and 16 . This result demonstrates that Platform A SoCs are more prone to critical errors considering the number of BBs on the output. Platform A SoCs have an average Diff BBs value of 1.98, more than double that of Platform $B$ SoCs, which is 0.85 . Figure ${ }^{-} 4$ also shows that it is more likely for radiation to corrupt HOG in a way that increase the number of BBs. In fact, only in $4 \%$ of Platform A corruptions and 5\% of Platform B corruptions Diff_BBs $<0$. This results is of extreme importance to describe HOG reliability. In fact, 


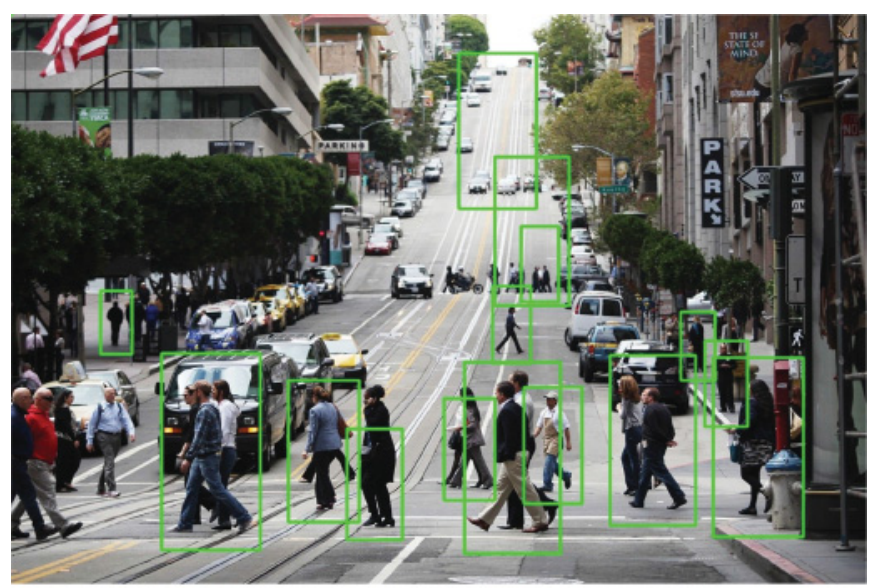

(a)

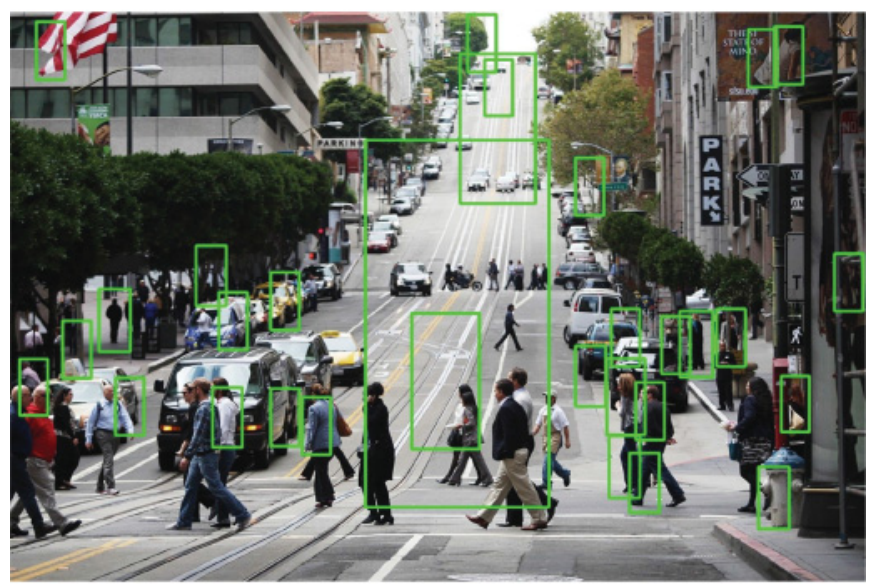

(b)

Fig. 4. Example of an experimentally observed error on a Platform A in which Diff_BBs $=16$. Panel (a) is the gold output, while panel (b) is the produced output.

experimental results confirm, as analyzed in Section 2.1, that HOG is intrinsically more prone to be corrupted in a way that increases the number of BBs.

As discussed in Section 3, we cannot state a priori that Diff_BBs $=0$ is sufficient or necessary to ensure that HOG corruption was not critical, that is, detection is still fine. We need to consider also BBs' position (Center of Mass) as well as Precision and Recall. Figure 6 shows the Center of Mass values for Platform A and Platform B SoCs. It is clear from the picture that most of the errors do not significantly modify the Center of Mass for both platforms. However, Platform A experienced some critical errors that could undermine its reliability. Considering only values with $D i f f \_B B s=0$ (not shown separately), the Center of Mass is never more than 1\% dislocated from the correct value. Only thanks to our experimental evaluation we can then conclude that when radiation corruption preserves the number of $\mathrm{BBs}$, the $\mathrm{BBs}$ are also located in the correct position.

Figure 7(a) shows the values for Precision and Recall for both Platform A and Platform $B$ SoCs, obtained comparing the corrupted output with the radiation-free output. 


\section{Diff_BBs}

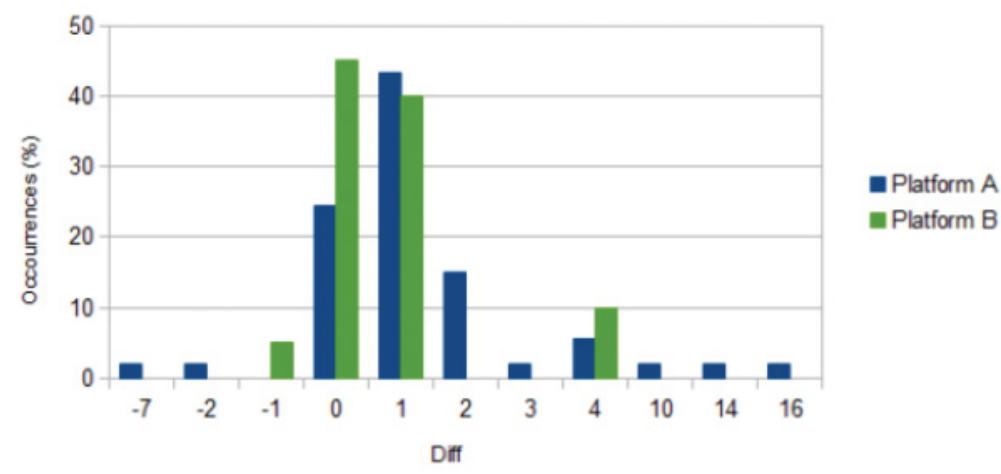

Fig. 5. Experimentally obtained Diff_BBs values for Platform A and Platform B SoCs. Only corrupted outputs are considered.

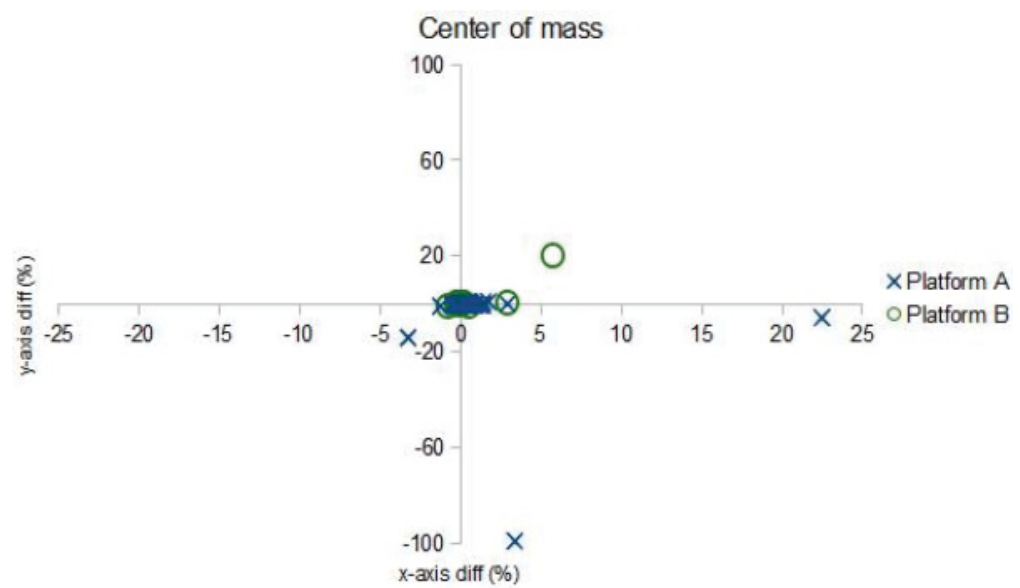

Fig. 6. Experimentally obtained Center of Mass values for Platform A and Platform B SoCs. The center of the Cartesian axes is the center of mass in the expected output. Only corrupted outputs are considered.

Most of the values for both Precision and Recall are concentrated on the top-right side of Figure 7(a). To better visualize the distribution for high values of Precision and Recall, Figure 7(b) depicts only Precision and Recall values greater than 90\%. As discussed in Section 3, Precision is the fraction of retrieved instances that are relevant. If Precision is lower than $100 \%$, then some detections are not relevant, that is, radiation leads HOG to mark as a pedestrian something it would not have marked without radiation. Having a low Precision value is risky in the sense that the system may trigger the vehicle to stop without apparent reason. Recall is the fraction of pedestrians that are actually detected in the corrupted output. If Recall is lower than $100 \%$, then some pedestrians HOG would normally detect are not detected because of radiation corruption. Low values for Recall are critical, as they could lead to undetected pedestrians and even to accidents if used in self-driving cars. In only $24 \%$ of corrupted executions for Platform A and $40 \%$ for Platform B SoCs, both Precision and Recall are preserved to $100 \%$ (values overlap in the single spot for Precision $=100 \%$ and Recall $=100 \%$ in both Figure 7(a) and Figure $7(\mathrm{~b})$ ). For those executions, HOG radiation corruption is not critical, as the error was insufficient to modify any BB in a way that refutes Equation (3). We recall 


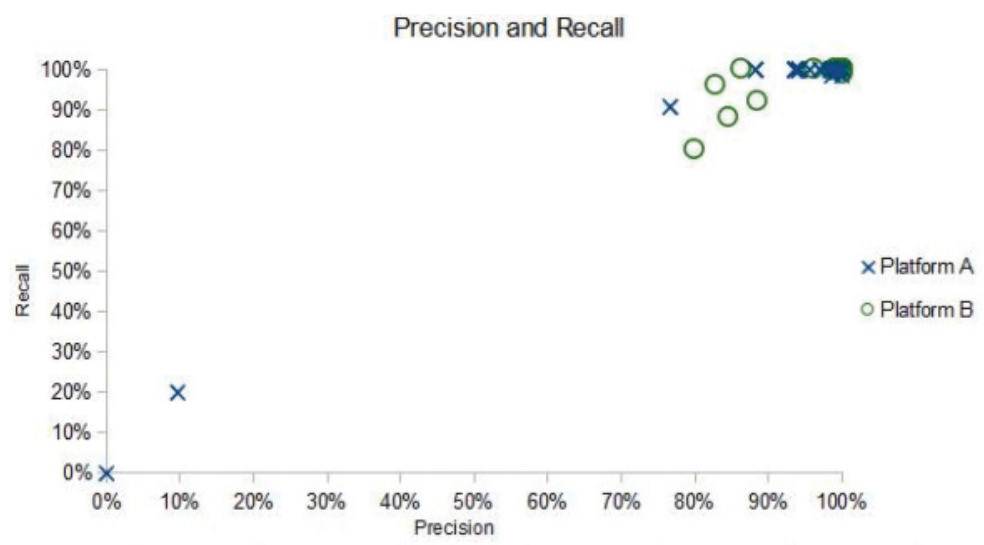

(a)

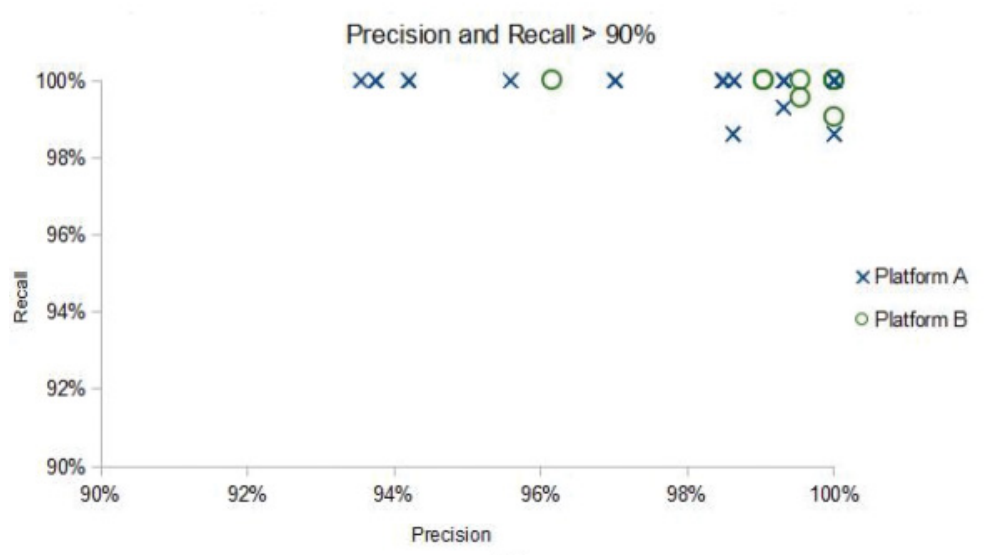

(b)

Fig. 7. A and Platform B experimentally obtained values for precision and recall. Values are calculated comparing corrupted outputs with the radiation-free output (not with the ground truth). (a) All values for precision and recall. (b) Precision and recall $>90 \%$. Twenty-four percent of values for Platform A and $40 \%$ for Platform $B$ overlap in the spot for precision $=100 \%$ and recall $=100 \%$.

that $T_{J}$ in Equation (3) is arbitrary. Results presented here are obtained with $T_{J}=0.5$, which derives from detection quality evaluations presented in Fawcett [2006]. Higher values for $T_{J}$ are likely to increase the number of radiation errors identified as critical. We made our experimental results available in a publicly accessible repository at dos Santos et al. [2016] to allow third-party analysis with different values for $T_{J}$.

Figure 7(a) shows that some rare errors cause Platform A Precision and Recall to be lower than $20 \%$ or even close to $0 \%$. We can tag those events as extremely critical as the corrupted output has little (if no) correlation with the expected value. As shown in Figure 7(a) and Figure 7(b), most radiation corruptions for both Platform $A$ and Platform B SoCs show both Precision and Recall higher than 90\%. However, we believe that while Precision can be relaxed, Recall should be maintained at $100 \%$. In fact, a non-relevant detection (i.e., Precision $<100 \%$ ) may at most cause an unnecessary stop while a miss-detection (i.e., Recall $<100 \%$ ) could lead to collision and eventual human life risk. If we conservatively accept Precision $\geq 90 \%$ but still request Recall $=100 \%$, then $85.1 \%$ of Platform $A$ and $65 \%$ of Platform $B$ corrupted executions would not be 


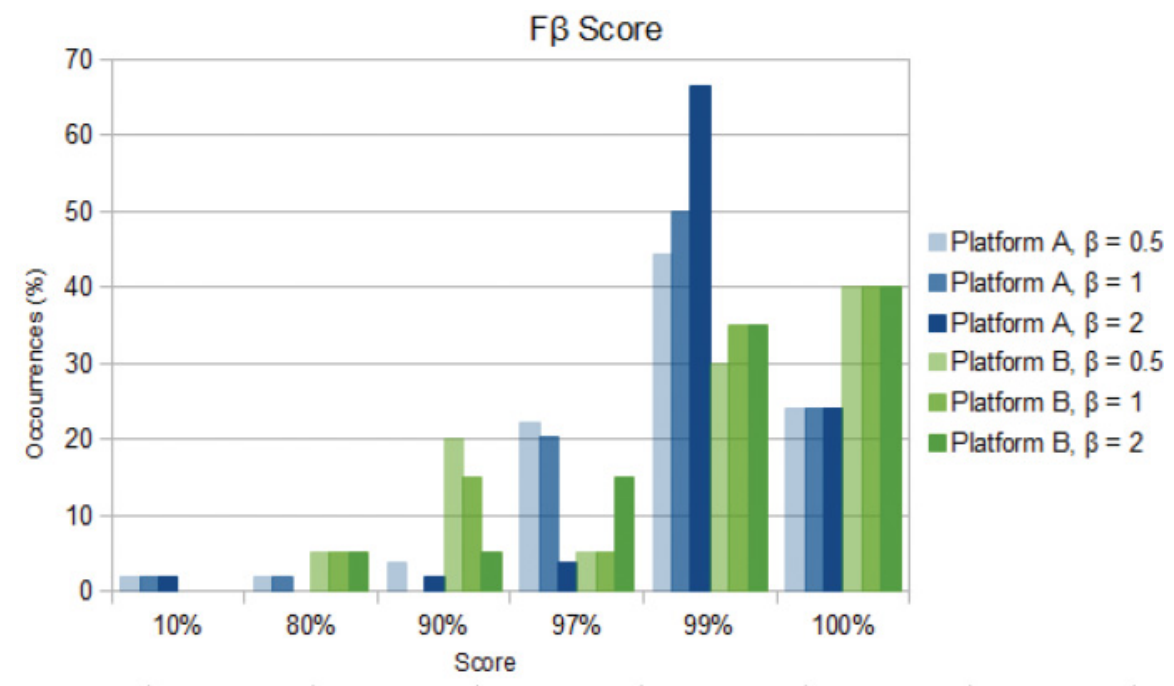

Fig. 8. $\quad$ F_ $\beta$ score with different values for Platform $A$ and Platform $B$. Only corrupted outputs are considered.

marked as critical. Under these circumstances, both the Platform $A$ and Platform $B$ SDC FIT rate would be significantly reduced, eventually being sufficient to be compliant with ASIL-D.

As expected, experimental results represented in Figure 7 show that radiationinduced errors are more likely to affect Precision than Recall. In fact, HOG corruption is more likely to increase the number of $\mathrm{BB}$ than reduce it. Additional BBs will affect Precision more than Recall. To confirm this intuition, we calculate the $F_{\beta}$ score for three values of $\beta$ (see Section 3 for details): (1) $\beta=0.5$ gives a higher weight to Precision, (2) $\beta=1$ considers Precision and Recall equally, and (3) $\beta=2$ gives a higher weight to Recall. As shown in Figure 8, the $F$-score values increase (indicating higher reliability) for increasing values of $\beta$. For $\beta=0.5$, Platform $A$ and Platform $B$ averaged scores of $94.4 \%$ and $96.1 \%$, respectively. For $\beta=1$, these values increase to $95.1 \%$ and $96.7 \%$. Finally, for $\beta=2$, the average $F_{\beta}$ is $95.9 \%$ for Platform $A$ and $97.3 \%$ for Platform $B$. This means that giving more importance to Recall, the reliability of HOG is increased. As Recall attests how many pedestrians were detected, we believe this to be an encouraging result.

To conclude our experimental data analysis, we apply Precision and Recall only to those corrupted results for which Diff_BBs $=0$ (not shown separately). We found that more than $90 \%$ of radiation-induced errors that do not affect the number of detected BBs let both Precision and Recall values be $100 \%$. We can then conclude that if the number of BBs is not affected by radiation, then the BBs are correctly positioned and have the expected area. Diff $B B s=0$ is then a sufficient condition for correct detection even in the event of failures. Those errors for which Diff $B B s=0$ are then not to be considered as critical for automotive applications. Analysis on our results affirm that Diff_BBs $\neq 0$ is necessary for the output to be considered critical but not sufficient. Outputs in which the radiation-induced error did not affect the number of BBs are wrong in very minor ways and can be considered correct, while those that had the number of BBs affected could potentially be critical and result in accidents.

\subsection{Fault-Injection Results}

Following the procedures described in Section 4.4, we have injected faults both on the host and GPU parts of HOG. We recall that failures were injected at execution 


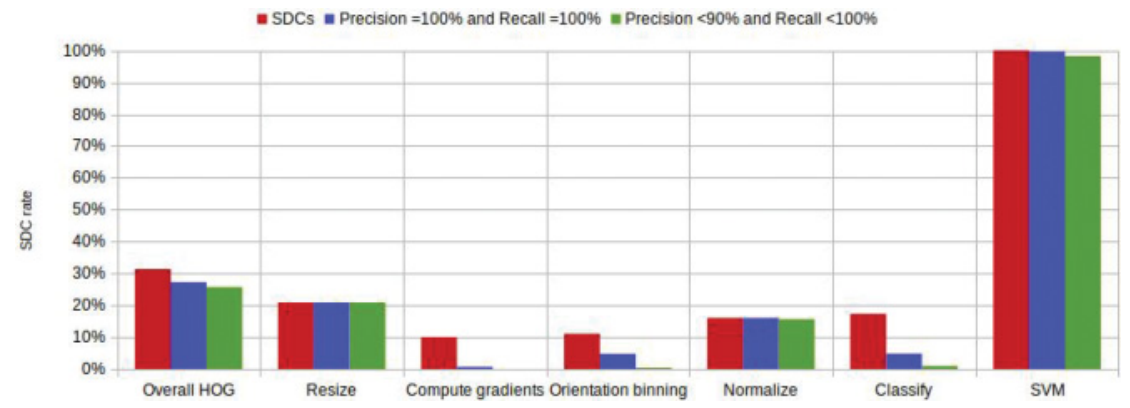

Fig. 9. Fault-injection and SDC rate for HOG and its kernels.

time only on the user-accessible resources through GDB. While impossible to be used as a comparison with radiation experiments, these data help us to understand the most critical portion of HOG. Figure 9 shows our results obtained with fault-injection on both CPU and GPU kernels (listed and detailed in Section 2.1). For each kernel of the algorithm (and for the overall HOG), we report the SDC rate, which is the percentage of injected faults that caused an observable error. As such, the SDC rate is an indication of how many radiation-induced errors affect computation. The higher the SDC rate, the more likely a radiation-induced error in the procedure is to affect HOG execution. It is worth noting that a procedure having a huge SDC rate does not mean that most radiation-induced failures observed during radiation test were caused by that procedure. In fact, SDC rate does not include information about the amount or sensitivity of resources involved in computation but only about their criticality.

Following the discussion in Section 5.2, we also distinguish between critical and noncritical errors. In Figure 9, we report also the SDC rate considering only injections that reduced Precision and Recall (Precision $<100 \%$ and Recall $<100 \%$ ) and that cause Precision to be lower than $90 \%$ and Recall to be lower than $100 \%$ (Precision < $90 \%$ and Recall $<100 \%$ ).

The SDC rate for the overall HOG algorithm is $31 \%$, which means that only $31 \%$ of the injections in all the GPU and CPU procedures impact HOG execution. Such a low SDC rate was expected, as HOG is an image processing code. In accordance with radiation experiment results, not all output errors impact HOG detection capability. As shown, $27 \%$ of injections cause an output error that affects Precision and Recall (Precision < $100 \%$ and Recall $<100 \%$ in Figure 9$)$ and $25 \%$ of injections reduce Precision preserving Recall (Precision $<90 \%$ and Recall $<100 \%$ in Figure 9). Fault-injection confirms the trend observed with radiation experiment. However, for fault-injection, the portion of noncritical errors is lower than for radiation experiments. This is due to the fact that our injection is performed at a high level of abstraction.

As shown in Figure 9, there are some portions of HOG that are more prone to faults. The most critical kernels in HOG are Resize, Block normalization, and Classifier. Twenty percent of faults injected in Resize caused an SDC. Unfortunately, all the observed SDCs caused by Resize corruption are to be considered critical, as both Precision and Recall were always lower than $85 \%$. Interestingly, we discovered that all the SDCs in Resize were caused by faults injected in the color texture variable. Injections in the other variables of Resize did not produce any effect on HOG output. This result will be used in the next section to efficiently harden Resize.

Gradient Computation shows the lowest SDC rate and the most significant behavior. Results show that only $0.7 \%$ of SDCs could not satisfy the condition of having both Precision and Recall equal to 100\%, and no injection caused Precision to be lower than $90 \%$ with a Recall lower than $100 \%$ (i.e., the SDC rate is 0 ). The Gradient Computation 
is a discrete derivative mask applied pixel-by-pixel to the frame. Even if a bit-flip happens while calculating the gradient on a pixel, all neighbor pixels still have the correct value and little to no effect is expected in the output.

Orientation Binning is in charge of calculating the cells and their orientation. According to Figure 9, Orientation Binning is very robust, as its SDC rate is about 11\%. Only $4.7 \%$ of errors injected in Orientation Binning generate SDCs that make both Precision and Recall to be lower than $100 \%$. If we relax criticality to Precision $<90 \%$ and Recall $<100 \%$, then this percentage is reduced to $0.4 \%$. Most observed errors in this step are caused by injections that affect the cell dimensions computation, while errors in the weighted vote and in the gradient calculation hardly affected the output.

The most critical GPU kernel is Block normalization. A corruption in this kernel results in a faulty grouping of cells or a wrong contrast normalization. The subsequent phases of HOG will then work on wrongly grouped or wrongly normalized blocks, which leads to mis-detection. Block Normalization is then to be considered as a critical procedure. As shown in Figure 9, the SDC rate of Block Normalization is almost the same even if criticality is considered.

Classifier is a complex kernel. It is in charge of identifying pedestrians among objects. Classifier's SDC rate is $17 \%$, and most of SDCs produced by injection on Classifier are not critical. In fact, the SDC rate for Precision $<90 \%$ and Recall $<100 \%$ is only $0.9 \%$. However, some errors show an extremely low Precision (lower than 22\%) and should then be carefully treated.

The host portion of HOG is simple and composed of memory operations only. The corruption of image data to be uploaded to the GPU modifies at most the propriety of a single pixel (color or illumination). Such errors are likely not to affect HOG computation and are not reported in Figure 9. On the contrary, an error during the upload of the SVM matrix or an error on the SVM matrix data stored either on the GPU or on the CPU is extremely critical. This matrix is computed in a previous training phase, which we assume fault free. However, the SVM matrix data is stored in memory, and eventually cache, as it is continuously used as a reference during computation. As a result, even if the training phase was successful, the computed SVM matrix values are exposed to radiation and prone to be corrupted. In our fault-injection campaign, every time that we changed any position of the SVM matrix, an SDC occurred (SDC rate is $100 \%$ ). We injected faults on the SVM while on the host, prior to the upload to the GPU. Similar results are to be expected if corruption occurs on SVM data on the GPU. As it can be seen in Figure 9, injections on the SVM matrix were almost all very critical. It is reasonable to assume that the SVM matrix will be uploaded once and used as a reference for several frames. As such, SVM matrix errors become extremely critical for $\mathrm{HOG}$, as all frames processed after the SVM matrix corruption will be erroneously computed, resulting in SDCs.

\subsection{Discussion on Selective Hardening}

The experimental results reported in Section 5.1 confirmed that HOG, being a filter and processing images, is more prone to experience Crashes than SDCs. A Crash is an undesired event in safety-critical systems but, being detectable with a watchdog, it can also be easily handled. The only constraint for Crashes hardening in a realtime system, like pedestrian detection in automotive applications, is to guarantee that deadlines are still met. In other words, even if a Crash occurs it is essential for HOG to become operative in time to maintain FTTI sufficiently low to ensure safety (details in Section 4.2).

SDCs, by their nature, are very hard to be detected and could be more harmful than Crashes. In the automotive market, full hardware redundancy is to be considered too expensive. As HOG is to be included in a real-time system, full software redundancy 
Table I. Execution Time and Selective Duplication Overheads for Each HOG Phase. Hardening Values Are the Overhead Imposed by Duplication Applied Only to One HOG Phase in Relation to the Unhardened Version Total Execution Time. The - Symbol Indicates That No Hardening Was Needed

\begin{tabular}{lccc}
\hline Phase & $\begin{array}{c}\text { Execution time } \\
\text { percentage }\end{array}$ & $\begin{array}{c}\text { Hardening overhead } \\
\text { without ECC }\end{array}$ & $\begin{array}{c}\text { Hardening overhead } \\
\text { with ECC }\end{array}$ \\
\hline SVM Matrix & $0 \%$ & $12 \%$ & $0 \%$ \\
Resize & $5.56 \%$ & $0 \%$ & $0 \%$ \\
Gradient Computation & $9.78 \%$ & - & - \\
Orientation Binning & $47.81 \%$ & - & - \\
Block Normalization & $14.43 \%$ & $23.2 \%$ & $3.2 \%$ \\
Classifier & $22.42 \%$ & $49.6 \%$ & $8.6 \%$ \\
\hline Total & $100 \%$ & $84.8 \%$ & $11.8 \%$ \\
\hline
\end{tabular}

is also to be considered impractical due to its huge overhead [Mitra 2010]. Based on our analysis, which goes through both host and GPU sides, we can identify the critical HOG procedures to be hardened, improving HOG's reliability significantly and in an efficient way. There are several ways to harden a code or portions of a code [Mitra 2010]. The scope of this section is to identify where hardening strategies should be applied, not to actually propose a specific hardening solution. However, to give a reference and evaluate how our fault-injection results impact the hardening solution overhead, we apply duplication with comparison, as it has already been demonstrated to detect more than $90 \%$ of SDCs in GPUs [de Oliveira et al. 2016]. ECC reduces of about 1 order of magnitude the SDCs rate of GPUs [de Oliveira et al. 2016] and, as we show in this section, if available, then it could significantly reduce duplication overhead. In fact, when ECC is present there is no need to duplicate and check memory values but only operations and computations. In the following discussion, we evaluate the overhead of duplication applied to critical HOG procedures with or without ECC available. Table I reports the overhead imposed to HOG by the hardening (by duplication) of each phase. A value of $12 \%$ in Table I, as for the SVM matrix, means that HOG will run $12 \%$ slower if duplication is applied only to SVM matrix. The overhead is higher when ECC is not present because of memory checks, which are very time consuming.

The host performs mainly data movement operations. Among data to be uploaded to the GPU, only the SVM matrix is extremely critical. SVM data is used to compute each frame, so it is likely to be in the SoC caches. Including ECC on the caches is a promising solution to significantly reduce the probability of having SVM data corrupted. If the caches ECC is not available (like in the considered platforms), then data in the cache should be duplicated or, alternatively, to frequently flush the caches. The main memory, in fact, is typically less prone to be corrupted than cache and is protected with ECC [Baumann 2005; Kim et al. 2007]. If SVM data duplication and check for eventual mismatches is necessary, then an overhead of $12 \%$ will be imposed to HOG execution time (see Table I).

In our fault-injection campaign, we found that Resize is a critical procedure. However, the observed errors were caused only by corruptions in the color parameters variables. Resize takes only $5.56 \%$ of overall execution time. It is not necessary to duplicate the whole procedure, but only the color texture variable. The duplication of this single variable introduces a negligible overhead in the overall HOG execution time yet significantly improves its reliability.

Gradient Computation and Orientation binning represents almost $9.78 \%$ and $47.81 \%$ of overall execution time, respectively. Since these two kernels did not produce critical errors, their hardening is likely not to significantly improve reliability. Based on our analysis and hardening efforts, we do not recommended hardening Gradient Computation and Orientation binning. Considering that these two steps are responsible of 
almost $60 \%$ of all HOG processing time, the overhead introduced by a fault tolerance technique in Gradient Computation and Orientation binning would unnecessarily increase HOG execution time and power consumption. In particular, the duplication of matrixes used both as input and output in Gradient Computation and Orientation binning would be extremely time consuming, resulting in a $40.5 \%$ (5.4\% with ECC) and $71 \%$ (12\% with ECC) overhead, respectively. These values are not reported in Table I as, considering the overheads and the fact that these kernels produced no critical SDCs during our fault-injection campaign, we suggest that duplication should not be applied.

Block Normalization has been identified to be the most critical kernel. Based on our fault-injection campaign and hardening efforts, we suggest hardening on Block Normalization. Since this step takes only $14.43 \%$ of the overall execution time and almost all produced SDCS are critical, hardening is mandatory. We observed an overhead of $23.2 \%$ in HOG execution time when duplicating Block Normalization with no ECC protection. If ECC is available, then this value drops to a much lower $3.2 \%$, as memory elements do not need to be duplicated and checked.

The last step of HOG execution is the Classifier phase. In our experiments, this phase got, in general, acceptable values of Precision and Recall. However, some outliers could significantly (but rarely) impact HOG reliability. Other classifiers can be used instead of the SVM-based one we considered. We expect the classifier to be intrinsically a critical portion of HOG, as it decides whether an object detected by HOG is actually a pedestrian. It is then reasonable to believe that hardening the classifier is essential to ensure high reliability. In our hardening efforts, duplicating this kernel yields an overhead of $49.6 \%$ if the absence of ECC is assumed and $8.6 \%$ if ECC is present.

Overall, considering our hardening suggestions and based on our efforts, we obtained a hardened version of the algorithm that runs $84.8 \%$ slower if no ECC is present. It is worth noting that this overhead is considerably lower than the average slowdown imposed by a full algorithm duplication, which is about 150\% [de Oliveira et al. 2016]. As expected, ECC could significantly improve the reliability of HOG reducing the overhead imposed by duplication. In fact, as reported in Table I, duplication implemented on an ECC-protected device will impose an overhead of only $11.8 \%$ to HOG.

\section{CONCLUSION AND FUTURE WORK}

In this article, we propose the first experimental evaluation of HOG-based pedestrian detection reliability. Using metrics derived from the image processing community, we investigate the behaviors of the HOG feature and SVM classifier executed on embedded GPUs exposed to atmospheric-like neutrons.

As expected, HOG is pretty robust against SDC, while it experiences a lot of Crashes. Our analysis helps identify those errors that are critical for automotive applications, which are a small fraction of the total. While being rare, those errors could lead to accidents and thus should be carefully considered.

Fault-injection results highlight the most critical procedures for HOG. Those procedures are exactly the ones that should be hardened, eventually with duplications.

In the future, we plan to consider pedestrian detection implemented thorough deep learning and neural networks. While being more accurate, this approach is also likely to be more resilient. We are also considering the idea of having the network trained to detect radiation-induced errors and prevent collisions or unexpected stops.

\section{REFERENCES}

Advanced Micro Devices AMD. 2015. BIOS and kernel developer guide for AMD family $16 \mathrm{~h}$ models $00 \mathrm{~h}-0 \mathrm{Fh}$ processors. Technical doc 48751 Rev 3.03. 
ARM. 2016. ARM Cortex-A73 MPCore Processor Technical Reference Manual Revision: r0p2. Retrieved from http://infocenter.arm.com/help/topic/com.arm.doc.100048_0002_04_en/cortex_a73_trm_100048_ 0002_04_en.pdf.

M. Bagatin, S. Gerardin, A. Paccagnella, A. Visconti, L. Chiavarone, M. Calabrese, and C. D. Frost. 2014. Sensitivity of NOR flash memories to wide-energy spectrum neutrons during accelerated tests. In Proceedings of the 2014 IEEE International Reliability Physics Symposium. 5F.3.1-5F.3.6. DOI : http://dx.doi.org/10.1109/IRPS.2014.6861094

R. C. Baumann. 2005. Radiation-induced soft errors in advanced semiconductor technologies. IEEE Trans. Device Mater. Reliabil. 5, 3 (Sept. 2005), 305-316. DOI : http://dx.doi.org/10.1109/TDMR.2005.853449

G. Bradski. 2000. The open source computer vision (OpenCV) library. Dr. Dobb's J. Softw. Tools 1, 1 (2000), 1.

M. A. Breuer, S. K. Gupta, and T. M. Mak. 2004. Defect and error tolerance in the presence of massive numbers of defects. IEEE Design Test Comput. 21, 3 (May 2004), 216-227. DOI:http://dx.doi.org/ 10.1109/MDT.2004.8

George Candea and Armando Fox. 2001. Recursive restartability: Turning the reboot sledgehammer into a scalpel. In Proceedings of the 8th Workshop on Hot Topics in Operating Systems (HOTOS'01). IEEE Computer Society, Washington, DC, 125.

C. Constantinescu. 2002. Impact of deep submicron technology on dependability of VLSI circuits. In Proceedings of the International Conference on Dependable Systems and Networks, 2002 (DSN 2002). 205-209. DOI : http://dx.doi.org/10.1109/DSN.2002.1028901

N. Dalal and B. Triggs. 2005. Histograms of oriented gradients for human detection. In Proceedings of the IEEE Computer Society Conference on Computer Vision and Pattern Recognition, 2005 (CVPR'05), Vol. 1. 886-893 vol. 1. DOI : http://dx.doi.org/10.1109/CVPR.2005.177

D. A. G. de Oliveira, L. L. Pilla, T. Santini, and P. Rech. 2016. Evaluation and mitigation of radiationinduced soft errors in graphics processing units. IEEE Trans. Comput. 65, 3 (March 2016), 791-804. DOI : http://dx.doi.org/10.1109/TC.2015.2444855

Nathan DeBardeleben, Sean Blanchard, Laura Monroe, Phil Romero, Daryl Grunau, Craig Idler, and Cornwell Wright. 2013. GPU behavior on a large HPC cluster. In Proceedings of the 6th Workshop on Resiliency in High Performance Computing (Resilience) in Clusters, Clouds, and Grids in Conjunction with the 19th International European Conference on Parallel and Distributed Computing (Euro-Par 2013).

P. Dollar, C. Wojek, B. Schiele, and P. Perona. 2012. Pedestrian detection: An evaluation of the state of the art. IEEE Trans. Pattern Anal. Mach. Intell. 34, 4 (Apr. 2012), 743-761. DOI : http://dx.doi.org/10.1109/ TPAMI.2011.155

J. J. Dongarra, H. W. Meuer, and E. Strohmaier. 2013. National Highway Traffic Safety Administration report. Retrieved from https://www.nhtsa.gov.

J. J. Dongarra, H. W. Meuer, and E. Strohmaier. 2015. ISO26262 Standard. Retrieved from https://www. iso.org/obp/ui/\#iso:std:iso:26262:-1:ed-1:v1:en.

Fernando Fernandes dos Santos, Paolo Rech, Daniel Oliveira, Lucas Weigel, Philippe Navaux, Luigi Carro, and Claudio Rogito Jung. 2016. taco2016-log-data. Retrieved from https://github.com/UFRGSCAROL/taco2016-log-data.

European New Car Assessment Programme. 2012. Euro NCAP Rating Review, Report from the Ratings Group. Retrieved from http://www.euroncap.com.

B. Fang, K. Pattabiraman, M. Ripeanu, and S. Gurumurthi. 2014. GPU-qin: A methodology for evaluating the error resilience of GPGPU applications. In Proceedings of the 2014 IEEE International Symposium on Performance Analysis of Systems and Software (ISPASS). 221-230. DOI:http://dx.doi.org/ 10.1109/ISPASS.2014.6844486

Tom Fawcett. 2006. An introduction to ROC analysis. Pattern Recogn. Lett. 27, 8 (2006), 861-874.

A. D. Fogle, Don Darling, R. C. Blish, and E. Daszko. 2004. Flash memory under cosmic and alpha irradiation. IEEE Trans. Device Mater. Reliabil. 4, 3 (Sept. 2004), 371-376. DOI:http://dx.doi.org/ 10.1109/TDMR.2004.834054

L. A. Bautista Gomez, Franck Cappello, L. Carro, N. DeBardeleben, B. Fang, S. Gurumurthi, K. Pattabiraman, R. Rech, and M. Sonza Reorda. 2014. GPGPUs: How to combine high computational power with high reliability. In Proceedings of the 2014 Design Automation and Test in Europe Conference and Exhibition. Dresden, Germany.

Google. 2016a. Google Self-Driving Car Project Montly Report. Retrieved June 1, 2016, from https://www. google.com/selfdrivingcar/reports/.

Google. 2016b. Wired Google's Self-Driving Car Caused Its First Crash. Retrieved April 5, 2016, http://www. wired.com/2016/02/googles-self-driving-car-may-caused-first-crash/. 
S. Hari, R. Venkatagiri, S. Adve, and H. Naeimi. 2014. TGangES: Gang error simulation for hardware resiliency evaluation. In Proceeding of the 41st Annual International Symposium on Computer Architecuture (ISCA'14).

John L. Hennessy and David A. Patterson. 2011. Computer Architecture, Fifth Edition: A Quantitative Approach (5th ed.). Morgan Kaufmann Publishers Inc., San Francisco, CA.

I. Herrera-Alzu and M. Lopez-Vallejo. 2014. System design framework and methodology for Xilinx virtex FPGA configuration scrubbers. IEEE Trans. Nucl. Sci. 61, 1 (Feb. 2014), 619-629. DOI:http://dx. doi.org/10.1109/TNS.2013.2292816

Intel. 2014. Intel Xeon Phi Coprocessor System Software Developers Guide. (2014). Retrieved from https://software.intel.com/sites/default/files/managed/09/07/xeon-phi-coprocessor-system-softwaredevelopers-guide.pdf.

Intel. 2016. Intel 64 and IA-32 architectures software developers manual combined volumes: 1, 2A, 2B, 2C, 2D, 3A, 3B, 3C and 3D. Retrieved from http://www.intel.com/content/dam/www/public/us/en/documents/ manuals/64-ia-32-architectures-software-developer-manual-325462.pdf.

JEDEC. 2006. Measurement and Reporting of Alpha Particle and Terrestrial Cosmic Ray-Induced Soft Errors in Semiconductor Devices. Technical Report JESD89A. JEDEC Standard.

T. Joachims. 1999. Making large-scale SVM learning practical. In Advances in Kernel Methods - Support Vector Learning, B. Schölkopf, C. Burges, and A. Smola (Eds.). MIT Press, Cambridge, MA, 169-184.

G. Just, J. L. Autran, S. Serre, D. Munteanu, S. Sauze, A. Regnier, J. L. Ogier, P. Roche, and G. Gasiot. 2013. Soft errors induced by natural radiation at ground level in floating gate flash memories. In Proceedings of the 2013 IEEE International Reliability Physics Symposium (IRPS). 3D.4.1-3D.4.8. DOI : http://dx.doi.org/10.1109/IRPS.2013.6531992

Minsung Kang and Young Chul Lim. 2014. Pedestrian detection using HOG-based block selection. In Proceedings of the 2014 11th International Conference on Informatics in Control, Automation and Robotics (ICINCO), Vol. 02. 783-787.

Saeng-Hwan Kim, Won-Oh Lee, Jung-Ho Kim, Seong-Seop Lee, Sun-Young Hwang, Chang-Il Kim, Tae-Woo Kwon, Bong-Seok Han, Sung-Kwon Cho, Dae-Hui Kim, Jae-Keun Hong, Min-Yung Lee, SungWook Yin, Hyeon-Gon Kim, Jin-Hong Ahn, Yong-Tark Kim, Yo-Hwan Koh, and Joong-Sik Kih. 2007. A low power and highly reliable 400Mbps mobile DDR SDRAM with on-chip distributed ECC. In Proceedings of the IEEE Asian Solid-State Circuits Conference, 2007 (ASSCC'07). 34-37. DOI : http://dx.doi.org/ 10.1109/ASSCC.2007.4425789

J. C. Laprie. 1995. Dependable computing and fault tolerance: Concepts and terminology. In Proceedings of the 25 International Symposium on Fault-Tolerant Computing, 1995, Highlights from Twenty-Five Years. 2. DOI : http://dx.doi.org/10.1109/FTCSH.1995.532603

K. Lee and L. Sha. 2005. Process resurrection: A fast recovery mechanism for real-time embedded systems. In Proceedings of the 11th IEEE Real Time and Embedded Technology and Applications Symposium. 292-301. DOI : http://dx.doi.org/10.1109/RTAS.2005.42

Man-Lap Li, Pradeep Ramachandran, Swarup Kumar Sahoo, Sarita V. Adve, Vikram S. Adve, and Yuanyuan Zhou. 2008. Understanding the propagation of hard errors to software and implications for resilient system design. SIGOPS Oper. Syst. Rev. 42, 2 (March 2008), 265-276. DOI:http://dx.doi.org/ $10.1145 / 1353535.1346315$

X. Li and X. Guo. 2013. A HOG feature and SVM based method for forward vehicle detection with single camera. In Proceedings of the 2013 5th International Conference on Intelligent Human-Machine Systems and Cybernetics (IHMSC), Vol. 1. 263-266. DOI:http://dx.doi.org/10.1109/IHMSC.2013.69

Joseph J. Lim, C. L. Zitnick, and Piotr Dollar. 2013. Sketch tokens: A learned mid-level representation for contour and object detection. In Proceedings of the IEEE Conference on Computer Vision and Pattern Recognition (CVPR).

R. R. Lutz. 1993. Analyzing software requirements errors in safety-critical, embedded systems. In Proceedings of IEEE International Symposium on Requirements Engineering, 1993. 126-133. DOI:http://dx. doi.org/10.1109/ISRE.1993.324825

Subhasish Mitra. 2010. System-Level Single-Event Effects. IEEE Nuclear and Space Radiation Effects Conference, NSREC 2012 Short Course. (2010).

Shubhendu S. Mukherjee, Christopher Weaver, Joel Emer, Steven K. Reinhardt, and Todd Austin. 2003. A systematic methodology to compute the architectural vulnerability factors for a high-performance microprocessor. In Proceedings of the 36th Annual IEEE / ACM International Symposium on Microarchitecture. IEEE Computer Society, Washington, DC, USA, 29-.

Nithin Nakka, Giacinto Paolo Saggese, Zbigniew Kalbarczyk, and Ravishankar K. Iyer. 2005. An Architectural Framework for Detecting Process Hangs / Crashes. Springer, Berlin, 103-121. DOI : http://dx.doi.org/ 10.1007/11408901_8 
M. Nicolaidis. 1999. Time redundancy based soft-error tolerance to rescue nanometer technologies. In Proceedings of the 17th IEEE VLSI Test Symposium, 1999. 86-94. DOI:http://dx.doi.org/ 10.1109/VTEST.1999.766651

Nvidia. 2014. Tegra K1 techinical reference manual. DP-06905-001-v03p (2014).

NVIDIA. 2015. NVIDIAs Next Generation CUDA Compute Architecture: Kepler GK110. Retrieved from http://www.nvidia.com/content/PDF/kepler/NVIDIA-Kepler-GK110-Architecture-Whitepaper.pdf.

Nvidia. 2016. Tegra X1 techinical reference manual. TRM DP 07225 001-v1.1p (2016).

D. A. G. Oliveira, P. Rech, H. M. Quinn, T. D. Fairbanks, L. Monroe, S. E. Michalak, C. Anderson-Cook, P. O. A. Navaux, and L. Carro. 2014. Modern GPUs radiation sensitivity evaluation and mitigation through duplication with comparison. IEEE Trans. Nucl. Sci. 61, 6 (Dec. 2014), 3115-3122. DOI : http://dx.doi.org/ 10.1109/TNS.2014.2362014

K. Pattabiraman, G. P. Saggese, D. Chen, Z. Kalbarczyk, and R. K. Iyer. 2006. Dynamic derivation of application-specific error detectors and their implementation in hardware. In Proceedings of the 2006 6th European Dependable Computing Conference, 97-108. DOI : http://dx.doi.org/10.1109/EDCC.2006.9

P. Rech, T. D. Fairbanks, H. M. Quinn, and L. Carro. 2013. Threads distribution effects on graphics processing units neutron sensitivity. IEEE Trans. Nucl. Sci. 60, 6 (Dec. 2013), 4220-4225. DOI:http://dx.doi.org/ 10.1109/TNS.2013.2286970

Xiaofeng Ren and Deva Ramanan. 2013. Histograms of sparse codes for object detection. In Proceedings of the IEEE Conference on Computer Vision and Pattern Recognition (CVPR).

G. P. Saggese, N. J. Wang, Z. T. Kalbarczyk, S. J. Patel, and R. K. Iyer. 2005. An experimental study of soft errors in microprocessors. IEEE Micro 25, 6 (Nov. 2005), 30-39. DOI :http://dx.doi.org/ 10.1109/MM.2005.104

Nirmal R. Saxena. 2016. Autonomous car is the new driver for resilient computing and design-for-test. In Silicon Errors in Logic-System Effects (SELSE) Keyonte. Retrieved from http://www.selse.org.

Bianca Schroeder, Eduardo Pinheiro, and Wolf-Dietrich Weber. 2009. DRAM errors in the wild: A large-scale field study. In SIGMETRICS.

Norbert Seifert, Xiaowei Zhu, and Lloyd W. Massengill. 2002. Impact of scaling on soft-error rates in commercial microprocessors. IEEE Trans. Nucl. Sci. 49, 6 (2002), 3100-3106.

S. Sivaraman and M. M. Trivedi. 2013. Looking at vehicles on the road: A survey of vision-based vehicle detection, tracking, and behavior analysis. IEEE Trans. Intell. Transport. Syst. 14, 4 (Dec 2013), 17731795. DOI : http://dx.doi.org/10.1109/TITS.2013.2266661

Jingweijia Tan, N. Goswami, Tao Li, and Xin Fu. 2011. Analyzing soft-error vulnerability on GPGPU microarchitecture. In Proceedings of the 2011 IEEE International Symposium on Workload Characterization (IISWC). 226-235. DOI : http://dx.doi.org/10.1109/IISWC.2011.6114182

M. Violante, L. Sterpone, A. Manuzzato, S. Gerardin, P. Rech, M. Bagatin, A. Paccagnella, C. Andreani, G. Gorini, A. Pietropaolo, G. Cardarilli, S. Pontarelli, and C. Frost. 2007. A new hardware/software platform and a new 1/E neutron source for soft error studies: Testing fpgas at the ISIS facility. IEEE Trans. Nucl. Sci. 54, 4 (2007), 1184-1189. DOI : http://dx.doi.org/10.1109/TNS.2007.902349

Chin-Hsien Wu, Tei-Wei Kuo, and Li-Pin Chang. 2006. The design of efficient initialization and crash recovery for log-based file systems over flash memory. Trans. Stor. 2, 4 (Nov. 2006), 449-467. DOI : http://dx.doi.org/ $10.1145 / 1210596.1210600$

H. J. Wunderlich, C. Braun, and S. Halder. 2013. Efficacy and efficiency of algorithm-based fault-tolerance on GPUs. In Proceedings of the 2013 IEEE 19th International On-Line Testing Symposium (IOLTS). 240-243. DOI : http://dx.doi.org/10.1109/IOLTS.2013.6604090

Xiaodong Yang, Chenyang Zhang, and YingLi Tian. 2012. Recognizing actions using depth motion mapsbased histograms of oriented gradients. In Proceedings of the 20th ACM International Conference on Multimedia (MM'12). ACM, New York, NY, 1057-1060. DOI : http://dx.doi.org/10.1145/2393347.2396382

Qiang Zhu, Mei-Chen Yeh, Kwang-Ting Cheng, and S. Avidan. 2006. Fast human detection using a cascade of histograms of oriented gradients. In Proceedings of the 2006 IEEE Computer Society Conference on Computer Vision and Pattern Recognition, Vol. 2. 1491-1498. DOI : http://dx.doi.org/10.1109/CVPR.2006.119

Received June 2016; revised August 2016; accepted September 2016 